\title{
Simulation of material budget measurements with beam telescopes
}

\author{
Paul Schütze \\ DESY \\ E-mail: paul.schuetze@desy.de \\ Hendrik Jansen* \\ DESY \\ E-mail: hendrik.jansen@desy · de
}

\begin{abstract}
High-precision particle tracking devices allow for a two-dimensional analysis of the material budget distribution of, e.g., particle detectors and their periphery. These tracking devices, called beam telescopes, enable a precise measurement of the trajectory of charged particles with a position resolution of a few micrometer and an angular resolution of the order of a few ten microradian. In this contribution, the material budget of a structured aluminium cube is reconstructed from various estimators based on the deflection angles of simulated electron trajectories. Probing a target under various rotation angles enables a tomographic reconstruction of the target.

We discuss the performance of width estimators of the scattering angle distributions and their impact on the contrast and the resolution of the reconstructed two- and three-dimensional images. At a voxel size of $0.1 \mathrm{~mm} \times 0.1 \mathrm{~mm} \times 0.1 \mathrm{~mm}$, we reconstruct the material budget with a contrast to noise ratio of $5.6 \pm 0.2$ and an edge resolution of about $(70 \pm 10)$ micrometre.
\end{abstract}

The 26th International Workshop on Vertex Detectors

10-15 September, 2017

Las Caldas, Asturias, Spain

\footnotetext{
*Speaker.
} 


\section{Introduction}

Charged particles undergo multiple Coulomb scattering while traversing material. The distribution of the angle between the incoming and the outgoing trajectory, i.e. before and after the material, is characterised by the amount of material budget traversed. Molière [1] postulated a theory without empirical parameters to describe multiple scattering in arbitrary materials. Later, Gaussian approximations to the involved calculations of his theory have been developed e.g. by Highland [2] in order to simplify predictions on these angular distributions. The measurement of the width of such distributions offers to quantify the amount of material budget interfering with the particle. Furthermore, the position-resolved measurement of the scattering angle distribution enables a three-dimensional imaging of any object's material budget distribution [3].

For this contribution, simulations have been performed replicating a $5 \mathrm{GeV} / c$ electron beam traversing a EUDET-type beam telescope [4] and a structured aluminium cube serving as a sample under test. Electrons in the GeV-range serve as beam particles carrying enough momentum to traverse few millimetre thick targets whilst undergoing multiple Coulomb scattering in the target allowing for a precise measurement of the deflection. A track reconstruction is performed enabling the position-resolved computation of the scattering angle of beam particles at the target arising from the multiple scattering therein. Various estimators are constructed in order to measure the width of the angular distribution, preferably in a precise, robust and unbiased manner. As the angular distributions differ from a normal distribution and may show noticeable tails, we evaluate the impact of the choice of the estimator on the reconstructed image in terms of contrast and resolution of the reconstructed images.

\section{Simulation set-up}

For the simulation presented, the AllPix [5] detector simulation framework was used, which is based on Geant 4 libraries [6, 7]. The libraries feature realistic models of the interaction of a highenergy particle beam with matter including multiple Coulomb scattering processes. Furthermore, the AllPix framework allows for the definition of active sensor volumes emulating the response of particle detectors using a data-driven digitization process.

The simulation mimics a realistic set-up, as it can be realised at the DESY Test Beam Facility [8]. Six pixelated silicon sensor planes, representing the DATURA beam telescope [4], are positioned around a sample under test (SUT), cf. Fig. 1 (left). For every simulated particle traversing the pixel sensor planes, a sensor response is calculated. The simulated response consists of clusters of one or more registered pixels, depending on the impact position of the particle, and is trimmed to its measured response [9].

The simulated SUT, shown in Fig. 1 (right), is an aluminium cube with an edge length of $6 \mathrm{~mm}$, featuring a rectangular cut-out of $3 \mathrm{~mm} \times 3 \mathrm{~mm} \times 1.5 \mathrm{~mm}$ at the bottom side. Furthermore, squared and round holes ranging from $0.1 \mathrm{~mm}$ to $1 \mathrm{~mm}$ in size and diameter are added.

In the chosen configuration of the beam telescope, the sample is placed in the centre of the beam telescope, half way between plane 2 and plane 3 , as shown in Fig. 1 . The distance $\mathrm{d} z_{\text {SUT }}$ between the SUT and the neighbouring sensors is to be minimised for optimal pointing resolution at the sample, whereas the distance $\mathrm{d} z$ between two sensor planes is maximised for improved 

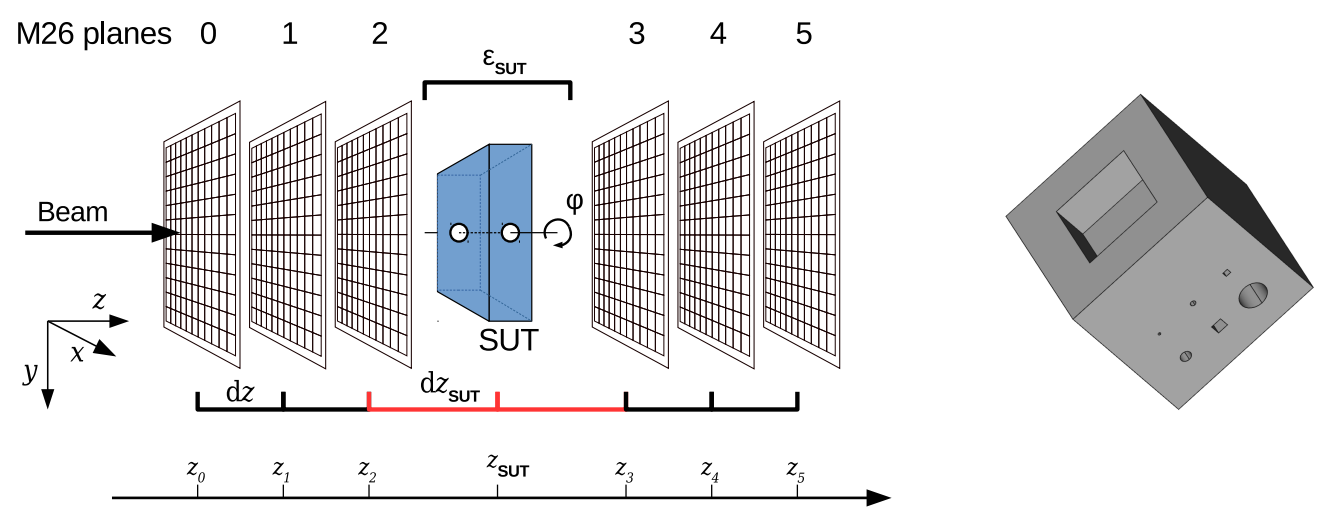

Figure 1: (left) Sketch of the DATURA beam telescope with its six pixel detector planes. The material budget image is reconstructed at a virtual $x$-y-plane at $z=z_{\text {SUT }}$. (right) The sample under test consists of a $6 \mathrm{~mm}$ aluminium cube with a rectangular cut-out as well as round and rectangular holes of different sizes.

angular resolution. Here, the distances were chosen to be $\mathrm{d} z_{\mathrm{SUT}}=10 \mathrm{~mm}$ and $\mathrm{d} z=150 \mathrm{~mm}$, again representing a realistic choice.

A simulated electron beam with a mean momentum of $5 \mathrm{GeV} / c$, a Gaussian momentum spread of $0.15 \mathrm{GeV} / c$, and a divergence of $1 \mathrm{mrad}$ illuminates the sample homogeneously. The Geant 4 model used ('emstandard') emulates, among other processes, energy losses due to ionisation and scattering processes [10]. Charged particles traversing any material are deflected by the electric field of the nuclei, resulting in an effective deflection and offset of the particle in the transverse plane when leaving the material, which depends on the traversed material budget $\varepsilon_{\text {SUT }}=d / X_{0}$ of the sample. The parameter $X_{0}$ denotes the radiation length of the material and is tabulated for various materials in reference [11], $d$ denotes the sample thickness. As an approximation to the Moliere theory, the central part of the distribution of scattering angles follows a normal distribution with the variance given by the Highland prediction [2,11]

$$
\theta_{0}^{2}=\left(\frac{13.6 \mathrm{MeV}}{\beta c p} \cdot z\right)^{2} \cdot \varepsilon \cdot(1+0.038 \cdot \ln (\varepsilon))^{2},
$$

with the particle's velocity $\beta c$, momentum $p$, charge number $z$ and the material budget $\varepsilon$. Hence the material budget and therefore the material properties of a sample can be extracted from a measurement of the scattering angle distribution.

\section{Material budget estimators}

The electron trajectories are reconstructed from their simulated sensor response using the EUTelescope track reconstruction framework $[12,13,14]$. The reconstruction method is described elsewhere [4, 15] and uses the concept of General Broken Lines [16, 17], taking into account the multiple Coulomb scattering in the sensor planes and in air. This results in precise knowledge of the incident position of the electron on the target and the deflection angle within the target.

In a virtual $x-y$-plane at the SUT, scattering angle distributions are created for cells of size $100 \mu \mathrm{m} \times 100 \mu \mathrm{m}$, adding up to 20000 cells for a field of view corresponding to the area of the 
(A)

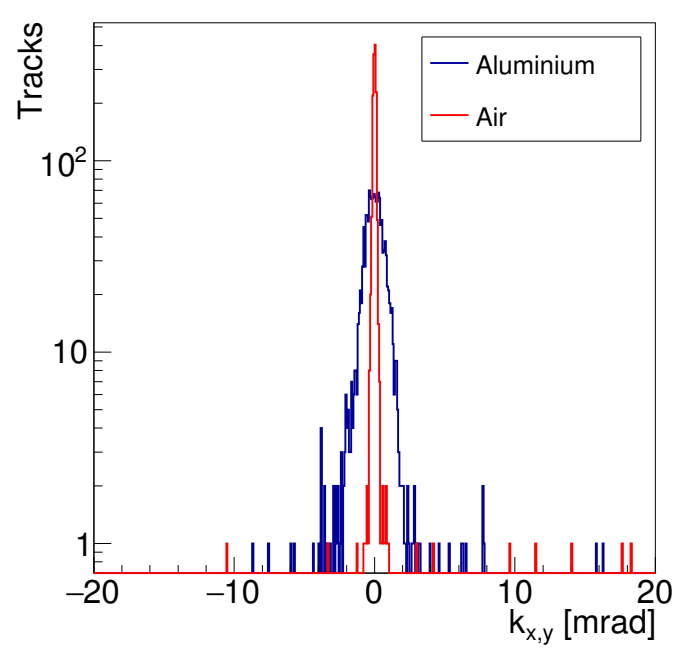

(B)

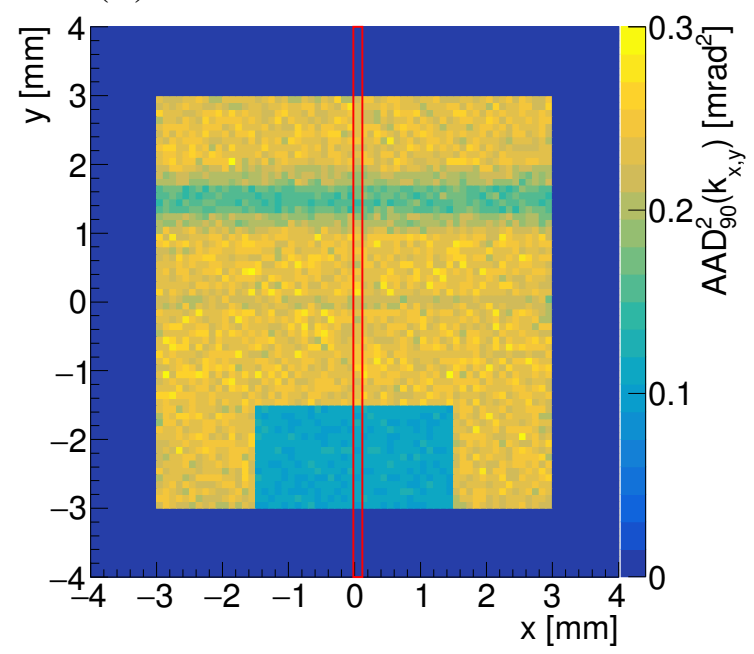

Figure 2: (A) For two given cells, one containing $6 \mathrm{~mm}$ aluminium, one containing air, the scattering angle distribution is shown combining the angles in the $x$ - and $y$-direction. (B) The square of the width extracted via the $\mathrm{AAD}_{90}$ estimator is shown within the transverse SUT plane. The red box indicates a data column subsequently used for image reconstruction.

Mimosa26 sensor. For each cell those tracks are considered, for which the reconstructed incident position falls within the cell. The average number of simulated tracks per cell for a two-dimensional image amounts to 1400 , the RMS of the number of tracks per cell is 70 . Two scattering angle distributions, one obtained from a cell containing $6 \mathrm{~mm}$ of aluminium with 1460 tracks and the other containing air only with 1386 tracks, are shown in figure 2 (A). From these distributions, the width $\theta_{0}$ - and the variance $\theta_{0}^{2}$ - is extracted for every cell. The number of tracks for a given cell at a given cell size fluctuates. Additionally, different estimation methods result in differing estimates of the width as they vary in sensitivity to the non-Gaussian part of the underlying distribution. Therefore, we construct various estimators of the width of the scattering angle distribution and compare their performance.

A priori, no absolute limit on the individual scattering angle is imposed in order not to bias the scattering angle distribution before extracting the width of the distributions. The following estimators are compared:

- RMS of the full data set $\left(\mathrm{RMS}_{100}\right)$, of the inner $95 \%$ quantile $\left(\mathrm{RMS}_{95}\right)$ and of the inner $90 \%$ quantile $\left(\mathrm{RMS}_{90}\right)$,

- Gaussian fit to the full data set (Gauss ${ }_{100}$ ) and to the inner 90\% quantile (Gauss95),

- Student's t plus Gauss fit to the full data set $\left(\mathrm{T}_{100}\right)$ and to the inner $90 \%$ quantile $\left(\mathrm{T}_{90}\right)$,

- Average absolute deviation of the full data set $\left(\mathrm{AAD}_{100}\right)$ and of the inner $90 \%$ quantile $\left(\mathrm{AAD}_{90}\right)$.

With the width extracted from every cell using the $\mathrm{AAD}_{90}$-estimator, an example of the two dimensional distribution of these widths at a sample rotation angle of $\varphi=90^{\circ}$ is shown in figure 2 (B). 
(A)

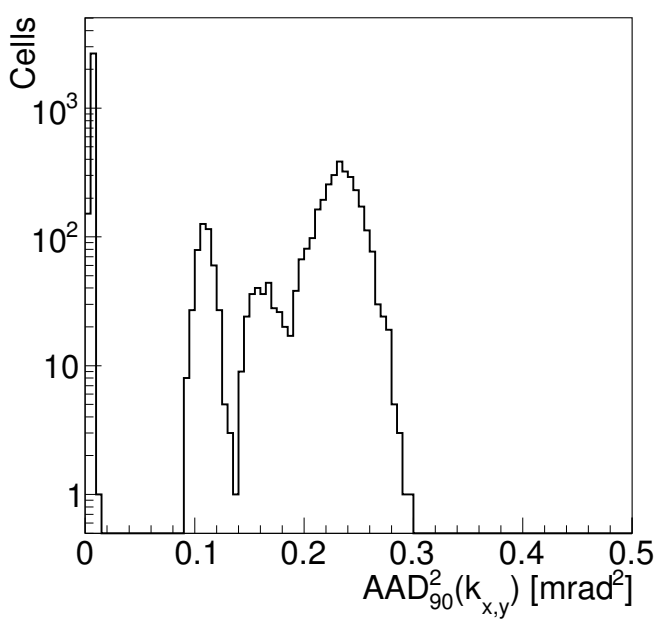

(B)

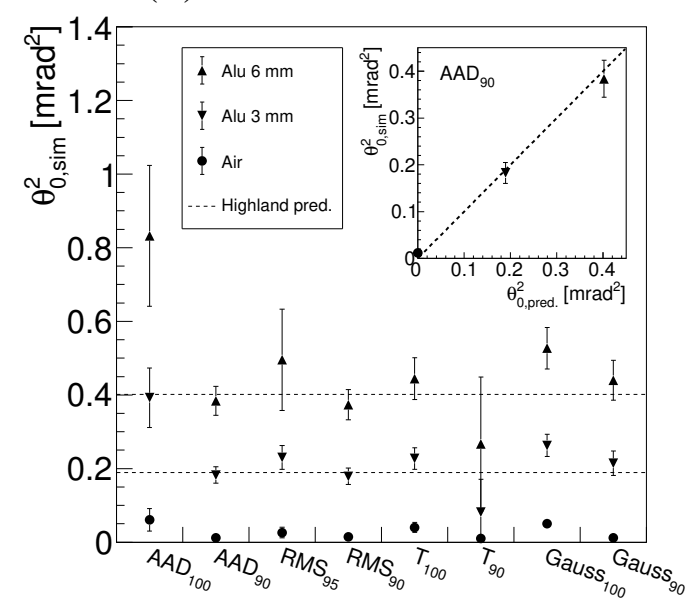

Figure 3: (A) The distribution of the squared widths for the $\mathrm{AAD}_{90}$ estimator is shown. (B) The mean of the squared widths and the RMS of the squared widths of various estimators are compared to the Highland prediction for air and two aluminium thicknesses. The horizontal lines represent the Highland predictions.

Clearly visible is the cut-out at the bottom and the regions with holes horizontally through the cube around $y=1.5 \mathrm{~mm}$ and less visibly at $y=0.0 \mathrm{~mm}$.

The histogram of the squared $\mathrm{AAD}_{90}$-widths is extracted from figure 2 (B) and shown in figure 3 (A). On the left-hand side, a sharp peak is formed from regions containing air (dark blue regions in figure $2(\mathrm{~B})$ ), the three peaks between 0.1 and $0.3 \mathrm{mrad}^{2}$ contain the values from regions with different thicknesses of aluminium. For three regions, i.e. air, $3 \mathrm{~mm}$ aluminium and $6 \mathrm{~mm}$ aluminium, the average (full circles and triangles) and the RMS (error bars) of the squared widths calculated from many cells are compared in figure 3 (B), with the various estimators listed along the $x$-axis. The results for $\mathrm{RMS}_{100}$ have been discarded from the graph owing to their very large error bars. The uncertainty decrease significantly for AAD and RMS when limiting the data set to the inner 90\% of the distribution, whereas it increases for the Student's t plus Gauss-fit and remains about constant for the Gauss fit. In order to be able to compare the central values with the Highland prediction, a scale factor $\sqrt{\pi / 2}$ has been applied to the AAD-values, which is the appropriate scale factor between the AAD and the standard deviation assuming a normal distribution. The performance of the estimators is judged from figure $3(\mathrm{~B})$, where two estimators, namely $\mathrm{AAD}_{90}$ and $\mathrm{RMS}_{90}$, present with the smallest RMS and an estimate for $\theta_{0}^{2}$ in good agreement with the Highland prediction. The inset of figure 3 (B) shows the linearity of the squared $\mathrm{AAD}_{90}$-estimator for different material budgets.

\section{Reconstructed tomographic image}

With the method described above, two-dimensional images as shown in figure 2 (B) are acquired that represent the position-resolved variance of the scattering angle distribution, and therefore an estimator for the material budget projected onto the $x-y$-plane, neglecting the logarithmic term in the Highland prediction, see eq. (2.1). To obtain information on the sample's inner 
(A)

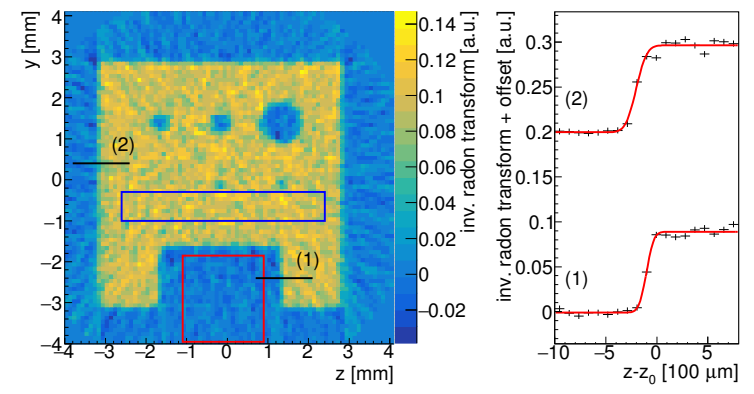

(B)

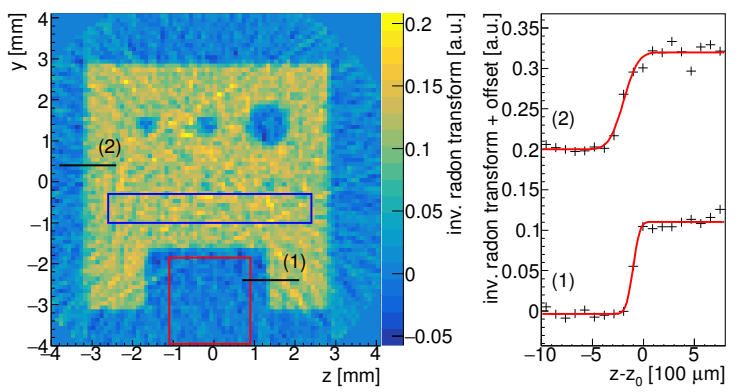

Figure 4: Contrast and edge resolution for the estimators (A) $\mathrm{AAD}_{90}$ and (B) $\mathrm{RMS}_{95}$. The coloured boxes indicate the regions used for the determination of the contrast, blue for aluminium, red for air. The edge resolution is evaluated at the two indicated positions.

structure, i.e. the material budget distribution along the $z$-axis, these images are split into vertical data columns, or slices, as indicated by the red box in figure 2 (B) and the simulation and analysis are repeated for different rotation angles of the sample. The corresponding vertical slices from 180 rotation angles with a step of $1^{\circ}$ are combined to form so-called sinograms. An inverse radon transform [18] of the sinogram of one slice yields a reconstruction of the two-dimensional material budget distribution in the $y$-z-plane. For this work, the open source software package scikit-image $[19,20]$ is used, which is capable of performing a filtered back projection based on the central-slice theorem. The procedure is described here in more detail [3]. At a voxel size of $100 \mu \mathrm{m} \times 100 \mu \mathrm{m} \times 100 \mu \mathrm{m}$, we reconstruct relative estimates of the material budget using two different estimators, $\mathrm{AAD}_{90}$ and $\mathrm{RMS}_{95}$, and compare their performance in terms of image contrast and image resolution.

Contrast The contrast to noise ratio (CNR)

$$
C N R=\frac{\mu_{\mathrm{alu}}-\mu_{\mathrm{air}}}{\sqrt{\sigma_{\mathrm{alu}}^{2}+\sigma_{\mathrm{air}}^{2}}}
$$

of the reconstructed image is defined as the ratio of the difference of the mean signal between aluminium and air of the inverse radon transform $(\mu)$ and the quadratic sum of the RMS of the signals $(\sigma)$. In figure 4 , the reconstructed image is shown for $\mathrm{AAD}_{90}(\mathrm{~A})$ and $\mathrm{RMS}_{95}(\mathrm{~B})$. The regions used to evaluate the CNR are marked with blue (aluminium) and red (air) rectangles. As expected, we find the image CNR is best for the estimator with the smallest variance in the input values, i.e. for $\mathrm{AAD}_{90}$. We report a CNR of $5.60 \pm 0.20$ using $\mathrm{AAD}_{90}$. For $\mathrm{RMS}_{95}$, i.e. an estimator with a larger spread from cell to cell, a CNR of $4.26 \pm 0.15$ is calculated.

Resolution The edge resolution, defined in reference [3], of the reconstructed image is evaluated and displayed for two estimators. In figure 4, the right-hand insets show the inverse radon transform values as a function of the depth coordinate for (A) $\mathrm{AAD}_{90}$ and (B) $\mathrm{RMS}_{95}$ at the positions indicated with (1) and (2), averaged over 12 voxels along the $y$-axis. The red lines represent error function fits to the simulation data. We find edge resolutions of $\sigma_{\text {edge }}=(67.9 \pm 7.5) \mu \mathrm{m}$ for $\mathrm{AAD}_{90}$ and $\sigma_{\text {edge }}=(78.4 \pm 10.4) \mu \mathrm{m}$ for $\mathrm{RMS}_{95}$. For sufficiently large contrast, the edge resolution is not 


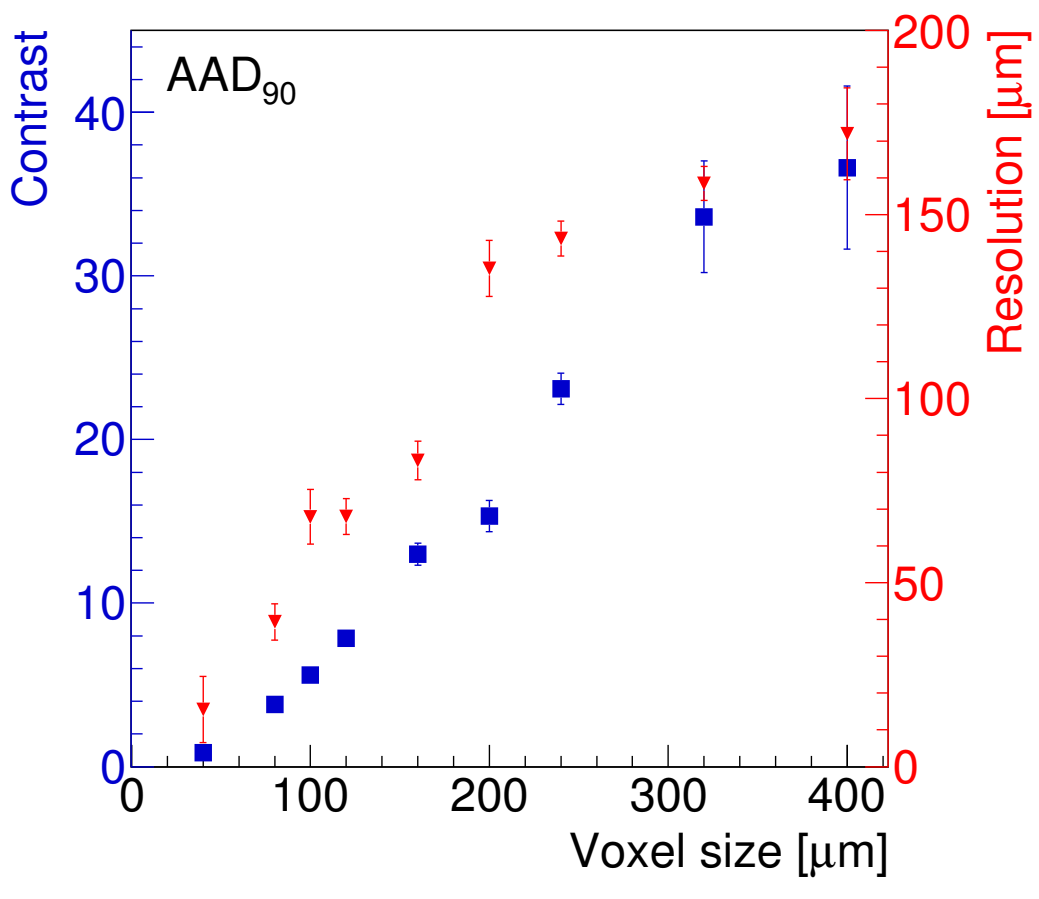

Figure 5: The image contrast and the edge resolution is shown for various voxel sizes for the $\mathrm{AAD}_{90^{-}}$ estimator.

affected strongly by the choice of the estimator. This is due to the fact, that at the chosen voxel size, the edge resolution is dominantly affected by the voxel size itself, with only one or two points in the critical region of the fit.

Resolution vs. contrast The methods explained above are repeated for various voxel sizes. Figure 5 shows the contrast (left $y$-axis) and the edge resolution (right $y$-axis) as a function of the cubic voxel size. With smaller voxel sizes the number of tracks per voxel decreases, increasing the image noise and hence decreasing the contrast. The image resolution improves, i.e. decreases, with smaller voxel sizes in the range studied herein. For the studied voxel sizes between $40 \mu \mathrm{m}$ and $400 \mu \mathrm{m}$, the extracted edge resolution is dominantly affected by the voxel size (about 0.5 times the voxel size). Therefore, the reported edge resolutions are rather upper limits on the achievable resolution. With both increasing contrast and edge resolution towards increasing voxel sizes, there is a trade-off between these two quantities when performing the image reconstruction.

\section{Conclusion}

We reported on a simulation of material budget measurements of an structured aluminium cube using $5 \mathrm{GeV} / c$ electrons and a beam telescope for tracking. Subsequent simulations for different rotation angles of the sample under test are used to reconstruct images along the depth of the sample. The contrast of the image reconstructed from the width of scattering angle distributions 
depends on the choice of the width estimator. The estimator with the smallest variance was found to be the average absolute deviation of the inner $90 \%$ quantile $\left(\mathrm{AAD}_{90}\right)$ resulting in an image $\mathrm{CNR}$ of $5.60 \pm 0.20$ at a voxel size of $100 \mu \mathrm{m} \times 100 \mu \mathrm{m} \times 100 \mu \mathrm{m}$. For this estimator, the voxel size was varied and the effect on the image resolution and image contrast was studied. Both the contrast and the edge resolution increase towards increasing voxel sizes.

\section{References}

[1] G. Moliere. Theorie der Streuung schneller geladener Teilchen II, Mehrfach- und Vielfachstreuung. Z. Naturforsch., 3a:78-97, 1948.

[2] V. Highland. Some practical remarks on multiple scattering. Nucl. Instrum. Methods Phys. Rev. A, 129(2):497-499, 1975.

[3] H. Jansen and P. Schütze. Feasibility of track-based multiple scattering tomography. Applied Physics Letters, 112(14):144101, 2018.

[4] H. Jansen et al. Performance of the EUDET-type beam telescopes. EPJ Techn. Instrum., 3(1):7, 2016. DESY-16-055, arXiv:1603.09669.

[5] M. Benoit, J. Idarraga, S. Arfaoui. AllPix Detector Simulation Framework. https://github. com/ALLPix/allpix. Accessed: 24.06.2017.

[6] S. Agostinelli et al. GEANT4 - a simulation toolkit. Nuclear Instruments and Methods in Physics Research Section A: Accelerators, Spectrometers, Detectors and Associated Equipment, 506(3):250 303, 2003.

[7] J. Allison et al. GEANT4 developments and applications. IEEE Transactions on Nuclear Science, 53(1):270-278, Feb 2006.

[8] Ralf Diener, Norbert Meyners, Natalia Potylitsina-Kube, and Marcel Stanitzki. Test Beams at DESY. http: / / testbeam. desy. de. Accessed: 26.07.2016.

[9] H. Jansen. Resolution studies with the DATURA beam telescope. J. Inst., 11(12):C12031, 2016.

[10] GEANT4 COLABORATION. Physics lists em constructors in geant4 10.4. http://geant4.cern.ch/collaboration/working_groups/electromagnetic/ physlist10.4.shtml.

[11] C. Patrignani et al. Review of Particle Physics. Chin. Phys., C40(10):100001, 2016.

[12] A. Bulgheroni et al. EUTelescope: Tracking Software. Technical report, 2007. Accessed: 21.04.2015.

[13] A. Bulgheroni et al. EUTelescope, the JRA1 Tracking and Reconstruction Software: A Status Report (Milestone). Technical report, 2008. Accessed: 21.04.2015.

[14] EUTelescope Software Developers. EUTelescope Website. http://eutelescope.desy.de. Accessed: 21.04.2015.

[15] Hendrik Jansen and Paul Schütze. Feasibility Study of a Track-based Multiple Scattering Tomography. In International Conference on Technology and Instrumentation in Particle Physics 2017, Beijing, under review.

[16] C. Kleinwort. General broken lines as advanced track fitting method. Nucl. Instr. Meth. Phys. Res. A, 673:107-110, May 2012. 
[17] V. Blobel, C. Kleinwort, and F. Meier. Fast alignment of a complex tracking detector using advanced track models. Computer Physics Communications, 182(9):1760 - 1763, 2011. Computer Physics Communications Special Edition for Conference on Computational Physics Trondheim, Norway, June 23-26, 2010.

[18] S.R. Deans. The Radon Transform and Some of Its Applications. Dover Books on Mathematics Series. Dover Publications, 2007.

[19] scikit-image development team. scikit-image. http://scikit-image.org/. Accessed: 24.06.2017.

[20] Stéfan van der Walt, Johannes L. Schönberger, Juan Nunez-Iglesias, François Boulogne, Joshua D. Warner, Neil Yager, Emmanuelle Gouillart, Tony Yu, and the scikit-image contributors. scikit-image: image processing in Python. PeerJ, 2:e453, 62014. 